\title{
A catalogue of OB associations in IC 1613
}

\author{
J. Borissova ${ }^{1}$, R. Kurtev ${ }^{2}$, L. Georgiev ${ }^{3}$, and M. Rosado ${ }^{3}$ \\ 1 Pontifica Universidad Catolíca de Chile, Facultad de Física, Departamento de Astronomía y Astrofísica, \\ Av. Vicuna Mackenna 4860, 782-0436 Macul, Santiago, Chile. Guest investigator of the UK Astronomy Data Centre \\ 2 Department of Astronomy, Sofia University and Isaac Newton Institute of Chile Bulgarian Branch, \\ James Bourchier Ave. 5, 1164 Sofia, Bulgaria \\ e-mail: kurtev@phys.uni-sofia.bg \\ ${ }^{3}$ Instituto de Astronomía, Universidad Nacional Autónoma de México, México \\ e-mail: [georgiev;margarit]@astroscu.unam.mx
}

Received 11 June 2001 / Accepted 15 September 2003

\begin{abstract}
We present a catalogue of OB associations in IC 1613. Using an automatic and objective method (Battinelli's 1991 technique) 60 objects were found. The size distribution reveals a significant peak at about 60 parsecs if a distance modulus of $24.27 \mathrm{mag}$ is assumed. Spatial distributions of the detected associations and H II regions are strongly correlated.
\end{abstract}

Key words. galaxies: individual: IC 1613 - galaxies: Local Group - galaxies: stellar content

\section{Introduction}

A stellar association is "a single, unbound concentration of early-type luminous stars, embedded in a very young starforming region" (Kontizas et al. 1999). The properties of the associations and the ionized gas clouds in which they are embedded allow tracing of the regions of most recent star formation in the galaxies. IC 1613 is a faint, irregular galaxy within the Local Group, which is resolvable into stars from the ground. The young stellar content of IC 1613 was investigated first by Hodge (1978). On the photographic plates taken with the 5-m Hale telescope he identified by eye estimates twenty OB associations. More recently, Freedman (1988) presented color-magnitude diagrams for 11 of them. Georgiev et al. (1999) presented the $U B V$ stellar photometry of the northeast sector of the galaxy. The properties of nine Hodge (1978) OB associations in this area are analyzed as well as seven new OB associations determined by cluster analysis. Valdez-Gutierrez et al. (2000) presented $\mathrm{H} \alpha$ and [SII] observations using the PUMA scanning Fabry-Perot interferometer. The kinematics of the ionized gas in the complex sample of nebulae was investigated. The ionized gas is distributed in classical H II regions and in a series of superbubbles. They found that almost every superbubble in the NE region has an interior association containing massive stars, suggesting a physical link between them. Rosado et al. (2001) continued the investigation of the influence of the massive stars in the interstellar medium of IC 1613 in the NW and southern region of the galaxy. In the southern region they found that the superbubbles

Send offprint requests to: J. Borissova, e-mail: jborisso@astro.puc.cl are probably formed by the winds of massive members of associations in spite of the close presence of a WO star. Lozinskaya et al. (2002) obtained spectra of the stars forming the chains and estimated their spectral types and luminosity classes. The stars were found to be at different evolutionary stages and six of them are identified as O stars. Lozinskaya et al. (2003) carried out detailed kinematical studies of the complex of multiple $\mathrm{H} \mathrm{I}$ and $\mathrm{H}$ II shells in region of ongoing star formation in the dwarf irregular galaxy IC 1613.

The purpose of the present paper, the fourth in our IC 1613 series, is to outline the new boundaries of the associations in IC 1613 using observational material of uniform quality and an objective method of identification of stellar associations.

\section{Observations and data reduction}

A set of $U B V$ frames of IC 1613 was obtained on the 2-m Richey-Chretien telescope of the Bulgarian National Astronomical Observatory "Rozhen" and the 2.1-m telescope of the San Pedro Martir observatory in Mexico. The exposure times of the images are between 600 and $1800 \mathrm{~s}$. The field of view of both telescopes is $5.6^{\prime} \times 5.6^{\prime}$. The seeing during these observations was $1-1.2^{\prime \prime}$ with stable and good photometric conditions. Three selected fields in the main body of the galaxy were observed. The Landolt (1992) standards were taken before and after all observations. The IRAF data reduction package was used to carry out the basic image reductions. The stellar photometry of the frames was performed with the point-spread function fitting routine ALLSTAR available in DAOPHOT (Stetson 1993). Complete details of the data reduction and analysis may be found in Georgiev et al. (1999). 
The zero-point errors of the transformations to the standard $U B V$ system are $0.04 \mathrm{mag}$. We transformed the $x, y$ coordinate systems of investigated fields to the one reference local system and then searched for stars in common between datasets. We check the residuals in magnitude and color for the stars in common. They do not show any systematic difference or trend so we can conclude that all datasets are homogeneous in magnitude within the errors. The artificial star technique (Aparicio \& Gallart 1995) was used to investigate crowding effects. The completeness can seriously affect our analysis for magnitudes fainter than $21 \mathrm{mag}$. There are no significant differences in the completeness factors between investigated fields. Since our analysis concerns only bright $\mathrm{OB}$ stars, no completeness correction was made. Field stars should not seriously affect the structure of the color-magnitude diagram because IC 1613 is situated far from the Galactic plane and no correction for contamination by field stars was applied. Fortunately IC 1613 has very low reddening - 0.03-0.06 (Freedman 1988; Georgiev et al. 1999), which also dos not affects our results.

\section{Associations}

The automated search for OB associations was carried out using Battinelli's (1991) algorithm (also called the PLC technique). The method assumes that "any given two stars in an ensemble of OB stars belong to the same association if and only if it is possible to connect these two stars, by successively linking $\mathrm{OB}$ stars located between them, separated from each other by no more than a certain fixed distance parameter, or search radius, called $d_{\mathrm{s}}$ ". The distance parameter $d_{\mathrm{s}}$ can be derived from the catalogue of early type stars using the function $f_{p}(d)$. This function describes the number of groups containing at least $p$ stars, for any given value of the distance parameter $d$. The function steeply rises until a maximum value is reached, followed by a gradual decrease towards a limiting value of 1 , which corresponds to the situation that the value of $d$ is so large that all stars can be assigned to one group. The optimum value of the distance parameter $d_{\mathrm{s}}$ is defined as the value of $d$ corresponding to the maximum of the function $f_{p}(d)$.

In order to select the catalogue of blue stars we need to adopt brightness and color cut-offs. In IC 1613 we selected as blue stars the stars that have absolute magnitudes $M_{V}<-2$ (assuming $(m-M)_{0}=24.27 \pm 0.1$, Dolphin et al. 2000) and dereddened colors $(B-V)_{0}<0.0$ (assuming $E(B-V)=0.03$, Rienke $\&$ Hodge 2001). Recently Pietrzynski et al. (2001) used the same algorithm to investigate associations in NGC 300. They adopted Battinelli's (1991) method adjusting the two important parameters in the Battinelli algorithm, described in the previous paragraph - distance parameter $d_{\mathrm{s}}$ and the minimum number of stars $p$ by numerical analysis. To derive the value of $d_{\mathrm{s}}$ Pietrzynski et al. (2001) applied the PLC technique with several values for the distance parameter and several values for the minimum number of stars, $p$. Their results show that the position of the maximum of the function $f_{p}(d)$ depends slightly on $p$ and is located in the relatively narrow interval (see their Fig. 2). The second parameter - the minimum number of stars $p$ of the potential association was determined by Pietrzynski et al. (2001) by performing the statistical tests. One hundred random distributions of a number of stars equal to the number of the sample of blue stars, and distributed over the same area, were created and the PLC technique was applied to search for potential groups. This experiment was repeated for different minimum numbers of stars. They found that the spurious detections are less than $10 \%$ if the six stars as a minimum population of OB stars of potential associations are chosen (see their Fig. 3).

Here, we follow Pietrzynski et al. (2001) approach for IC 1613. The value of $d_{\mathrm{s}}$ was derived applying the method with different values for the distance parameter. In our case the position of the maximum of the function $f_{p}(d)$ is located in the interval between 17 and 23 pixels. The number of associations obtained with $d_{\mathrm{s}}=17,18 \ldots 22$ and 23 pixels changes by less than 5\%. Thus we confirm the Pietrzynski et al. (2001) result that the number of detected OB associations is insensitive to the adopted value of the search radius in this interval. We adopted $d_{\mathrm{s}}=20$ pixels as a search radius. The next parameter that needs to be specified is the minimum number of stars $p$ of the potential association. Again, using the statistical approach of Pietrzynski et al. (2001), described in the previous paragraph we derive four OB stars as a minimum number of stars in some clump in order to have a "real" association.

With the above described parameters Battinelli's (1991) algorithm selects 58 associations with sizes between 30 and $130 \mathrm{pc}$ (with accepted distance of $730 \mathrm{kpc}$, Dolphin et al. 2000).

And finally to check our results and to cover the whole galaxy we retrieve the $U B V$ images of IC 1613 from the ING Archive, UK Astronomy Data Center, Cambridge. The images were obtained on 11 December 1998 with the 2.5-m Isaac Newton Telescope and Wide Field Camera (WFC), which has a field of view of $11.4 \times 22.8$ arcmin. Unfortunately, they are not of high quality and not as deep as Rozhen images. We reduced the images and transformed the instrumental magnitudes to the standard $U B V$ system using about 100 common stars between WFC and Rozhen photometry. Then we ran again Battinelli's (1991) algorithm in the same way as described above. On the WFC images we found 55 associations. Comparing with the associations obtained from Rohzen images there are 5 omitted associations (G1, G2, G4, G8 and G26, see Table 1) and 2 additional (G59 and G60) ones. The WFC images are less deep than Rozhen data by approx. 1 mag in $U$ and there are less than four OB stars in each of the omitted associations. Also, the boundaries of some associations in the fields in common are slightly different. In general the average quantities of the common associations in the two lists however are little affected. The two additional associations are found in the areas not covered by the Rozhen frames.

The map of the association boundaries resulting from the automated search of IC 1613 is shown in Fig. 1 and the properties of the associations are summarized in Table 1. Columns 1, 2 and 3 give the designation and the equatorial coordinates of the detected associations. The number of bright $\left(M_{V}<-2\right)$ OB members in each of the associations, their sizes in parsecs and cross-identification with the catalogue of Hodge (1978) are given in the next columns. The coordinates of the OB associations were determined as the mean coordinates of their separate member stars. 
Table 1. Catalogue of OB associations in IC 1613.

\begin{tabular}{|c|c|c|c|c|c|c|c|c|c|c|c|}
\hline Name & $\alpha_{2000}$ & $\delta_{2000}$ & $\operatorname{size}(p c)$ & OB stars & Hodge & Name & $\alpha_{2000}$ & $\delta_{2000}$ & $\operatorname{size}(p c)$ & OB stars & Hodge \\
\hline G1 & 01:04:53.92 & $+02: 10: 08.49$ & 33 & 5 & - & G32 & 01:04:53.04 & $+02: 06: 11.00$ & 60 & 7 & H9 \\
\hline G2 & 01:04:54.11 & $+02: 08: 50.65$ & 46 & 5 & - & G33 & 01:04:57.28 & $+02: 05: 15.55$ & 40 & 10 & H9 \\
\hline G3 & 01:04:54.84 & $+02: 10: 30.98$ & 35 & 5 & - & G34 & 01:04:59.08 & $+02: 04: 23.50$ & 60 & 6 & H9 \\
\hline G4 & 01:04:57.74 & $+02: 10: 56.39$ & 30 & 4 & - & G35 & 01:04:55.95 & $+02: 05: 44.97$ & 84 & 18 & $\mathrm{H} 9$ \\
\hline G5 & 01:04:58.04 & $+02: 08: 56.80$ & 27 & 5 & - & G36 & 01:05:00.54 & $+02: 05: 17.01$ & 57 & 7 & H9 \\
\hline G6 & 01:04:58.54 & $+02: 08: 22.14$ & 43 & 6 & - & G37 & 01:04:57.87 & $+02: 04: 46.41$ & 65 & 7 & H9 \\
\hline G7 & 01:04:59.49 & $+02: 09: 17.81$ & 129 & 36 & $\mathrm{H} 10$ & G38 & 01:05:00.47 & $+02: 05: 06.94$ & 79 & 13 & H9 \\
\hline G8 & 01:04:59.43 & $+02: 08: 40.54$ & 28 & 4 & - & G39 & 01:04:53.61 & $+02: 05: 44.15$ & 72 & 7 & $\mathrm{H} 8$ \\
\hline G9 & 01:05:00.26 & $+02: 10: 13.41$ & 33 & 4 & - & G40 & 01:04:59.36 & $+02: 05: 52.03$ & 45 & 6 & $\mathrm{H} 8$ \\
\hline G10 & 01:05:00.59 & $+02: 10: 42.84$ & 112 & 36 & H11 & G41 & 01:04:58.76 & $+02: 05: 24.97$ & 62 & 8 & $\mathrm{H} 8$ \\
\hline G11 & 01:05:00.91 & $+02: 09: 41.73$ & 92 & 14 & H13 & G42 & 01:04:53.07 & $+02: 04: 56.66$ & 64 & 4 & - \\
\hline G12 & 01:05:01.21 & $+02: 08: 33.03$ & 40 & 6 & H14 & G43 & 01:04:49.11 & $+02: 06: 15.83$ & 44 & 5 & - \\
\hline G13 & 01:05:01.64 & $+02: 07: 31.51$ & 42 & 6 & H14 & G44 & 01:05:01.78 & $+02: 06: 19.08$ & 42 & 6 & $\mathrm{H} 7$ \\
\hline G14 & 01:05:02.22 & $+02: 08: 44.37$ & 107 & 27 & H14 & G45 & 01:04:50.98 & $+02: 04: 29.19$ & 54 & 6 & - \\
\hline G15 & 01:05:02.22 & $+02: 08: 03.08$ & 112 & 50 & H15 & G46 & 01:04:48.86 & $+02: 06: 47.12$ & 76 & 8 & H6 \\
\hline G16 & 01:05:02.66 & $+02: 09: 25.29$ & 55 & 8 & H13 & G47 & 01:04:50.69 & $+02: 07: 01.43$ & 101 & 10 & H6 \\
\hline G17 & 01:05:03.05 & $+02: 09: 38.14$ & 31 & 7 & H13 & G48 & 01:05:02.28 & $+02: 06: 01.92$ & 106 & 9 & $\mathrm{H} 7$ \\
\hline G18 & 01:05:03.43 & $+02: 08: 35.60$ & 71 & 11 & $\mathrm{H} 14$ & G49 & 01:04:51.61 & $+02: 06: 23.82$ & 49 & 4 & - \\
\hline G19 & 01:05:04.17 & $+02: 10: 16.83$ & 51 & 6 & - & G50 & 01:04:46.46 & $+02: 07: 28.28$ & 55 & 4 & $\mathrm{H} 4$ \\
\hline G20 & 01:05:04.98 & $+02: 11: 55.88$ & 53 & 7 & H16 & G51 & 01:04:46.28 & $+02: 08: 59.07$ & 84 & 6 & $\mathrm{H} 4$ \\
\hline $\mathrm{G} 21$ & 01:05:04.77 & $+02: 09: 31.83$ & 103 & 23 & $\mathrm{H} 17, \mathrm{H} 13$ & G52 & 01:04:45.92 & $+02: 06: 28.20$ & 82 & 6 & H5 \\
\hline $\mathrm{G} 22$ & 01:05:05.16 & $+02: 11: 14.26$ & 86 & 10 & - & G53 & 01:04:41.69 & $+02: 09: 16.98$ & 112 & 10 & - \\
\hline G23 & 01:05:05.17 & $+02: 08: 50.48$ & 28 & 5 & $\mathrm{H} 17$ & G54 & 01:04:44.98 & $+02: 09: 24.31$ & 37 & 5 & - \\
\hline G24 & 01:05:06.07 & $+02: 09: 01.59$ & 45 & 5 & - & G55 & 01:04:33.46 & $+02: 08: 21.15$ & 80 & 6 & $\mathrm{H} 3$ \\
\hline G25 & 01:05:06.28 & $+02: 09: 30.08$ & 37 & 11 & H17 & G56 & 01:04:50.46 & $+02: 08: 14.68$ & 76 & 5 & $\mathrm{H} 3$ \\
\hline G26 & 01:05:06.26 & $+02: 06: 52.73$ & 40 & 5 & - & G57 & 01:04:43.40 & $+02: 08: 17.12$ & 76 & 5 & $\mathrm{H} 2$ \\
\hline G27 & 01:05:06.71 & $+02: 08: 39.35$ & 72 & 11 & - & G58 & 01:04:46.84 & $+02: 08: 17.81$ & 90 & 11 & $\mathrm{H} 1$ \\
\hline $\mathrm{G} 28$ & 01:05:07.59 & $+02: 09: 55.16$ & 111 & 15 & H17, H18 & G59 & 01:05:10.41 & $+02: 12: 27.30$ & 60 & 15 & $\mathrm{H} 20$ \\
\hline G29 & 01:05:10.05 & $+02: 10: 24.48$ & 68 & 8 & H19 & G60 & 01:04:26.1 & $+02: 06: 56.05$ & 28 & 4 & - \\
\hline G30 & 01:05:13.68 & $+02: 08: 22.22$ & 41 & 8 & - & & & & & & \\
\hline G31 & 01:04:54.60 & $+02: 06: 04.61$ & 52 & 6 & H9 & & & & & & \\
\hline
\end{tabular}

Comparison with the stellar associations outlined by Hodge (1978) shows that most of them coincide with our groups, but in general each Hodge association divides into several smaller groups. The "new" associations have smaller sizes and look like bright cores within Hodge associations. Hodge (1986) describes the problem of the different sizes of the stellar associations in the Magellanic clouds and other nearby galaxies as M31 and M33. He concluded that the mean size of the associations depends on the image scale and the distance to the galaxy. It is known also that young associations in the Galaxy, like the Trapezium, contain small compact subgroups similar to those selected in IC 1613 (Lada \& Lada 2003) while the older associations consist of randomly distributed stars and they could not be divided into subgroups. The Battinelli (1991) criterion obviously detects the youngest compact groups and this may explain the smaller mean size of the stellar groups identified in the present paper than those presented by Hodge (1978).

In Fig. 2 (top panel) we show the size distribution of the detected associations. There is a peak located at $60 \mathrm{pc}$. Comparing the IC 1613 associations with the Magellanic Clouds, M 31, M 33 and NGC 6822 (Bresolin et al. 1998; Ivanov 1996) associations we can see that the distribution of their sizes is similar with a peak between 40 and $80 \mathrm{pc}$. The mean size of the associations in IC 1613 is $63 \mathrm{pc}$ and is in agreement with the mean sizes of associations in LMC-60 pc, SMC-70 pc and M33-60 pc (Bresolin et al. 1998). All these measurements are performed using the same Battinelli (1991) method. In order to check whether these similar sizes are caused by the identification algorithm we performed a numerical analysis. Using DAOPHOT in IRAF we build the artificial images for our Field II. Field II contains 19 associations with 


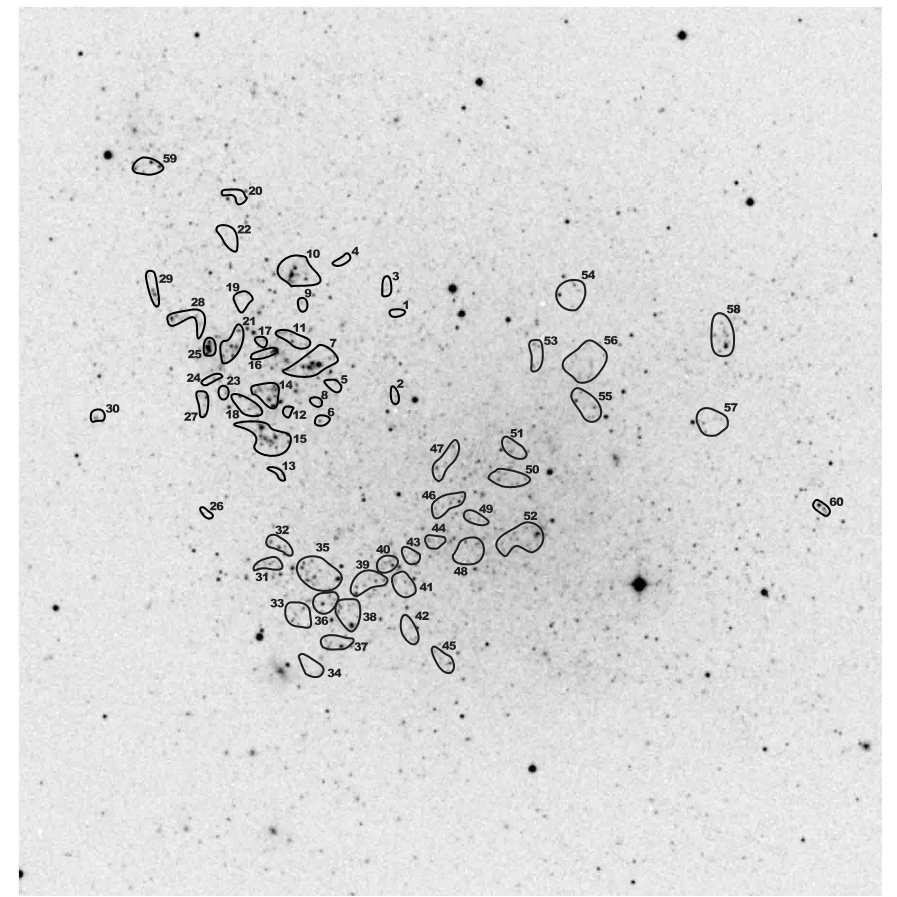

Fig. 1. Map of the associations in IC 1613 outlined by Battinelli (1991) algorithm.

a mean size of $64 \mathrm{pc}$ and an average of $7 \mathrm{OB}$ stars. To conserve the completeness we chose to add 3 artificial associations with 7 "OB" stars. We created these artificial associations with the PSF obtained for Field II and added them randomly to the image. We then applied Battinelli's (1991) algorithm. We repeat this procedure many times. As a result we found that the size of an association does not depend on how close the neighboring association is. The same procedure was used to estimate the spurious detections of the associations due to random concentrations of blue stars. Here, we artificially added 21 "OB" stars (10\% of the whole sample in Field II) at random positions and created 20 images. Again, we apply Battinelli's (1991) algorithm and estimated that between 3-5 OB associations in the whole sample could be randomly concentrated OB stars.

The histogram of the number of $\mathrm{OB}$ stars per association is shown in Fig. 2, bottom panel. As can be seen most of the associations have fewer than twelve OB members, with a maximum of the distribution at seven OB members. The most populous association contains $50 \mathrm{OB}$ stars.

\section{Stellar associations and superbubbles}

The associations are not uniformly distributed within the galaxy and they are mostly concentrated in the NE and NW regions (Hodge 1978). On the other hand, the ionized gas in IC 1613 is concentrated in large diameter, expanding superbubbles (Valdez-Gutierrez et al. 2000) in the same regions. Comparison with the velocity map of the superbubbles (Fig. 6 in Valdez-Gutierrez et al. 2000) shows that almost every superbubble in IC 1613 has an interior association. These associations are located within or at the periphery of the superbubbles
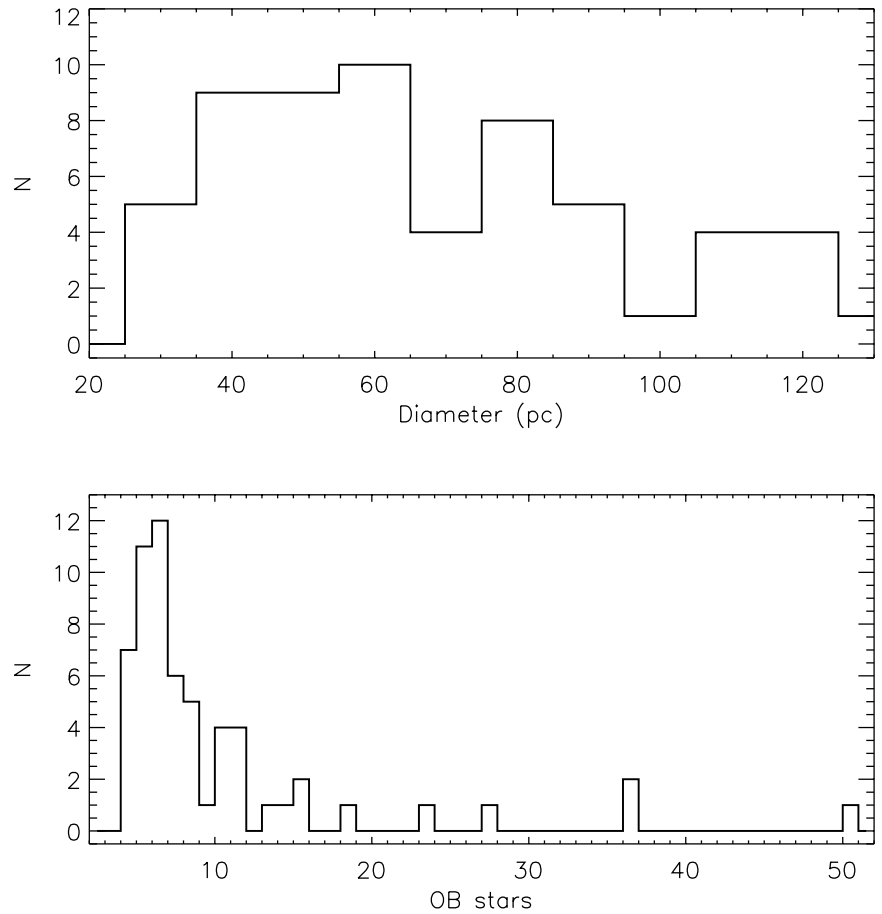

Fig. 2. Top panel: size distribution of associations in IC 1613. Bottom panel: histogram of the number of $\mathrm{OB}$ stars per association.

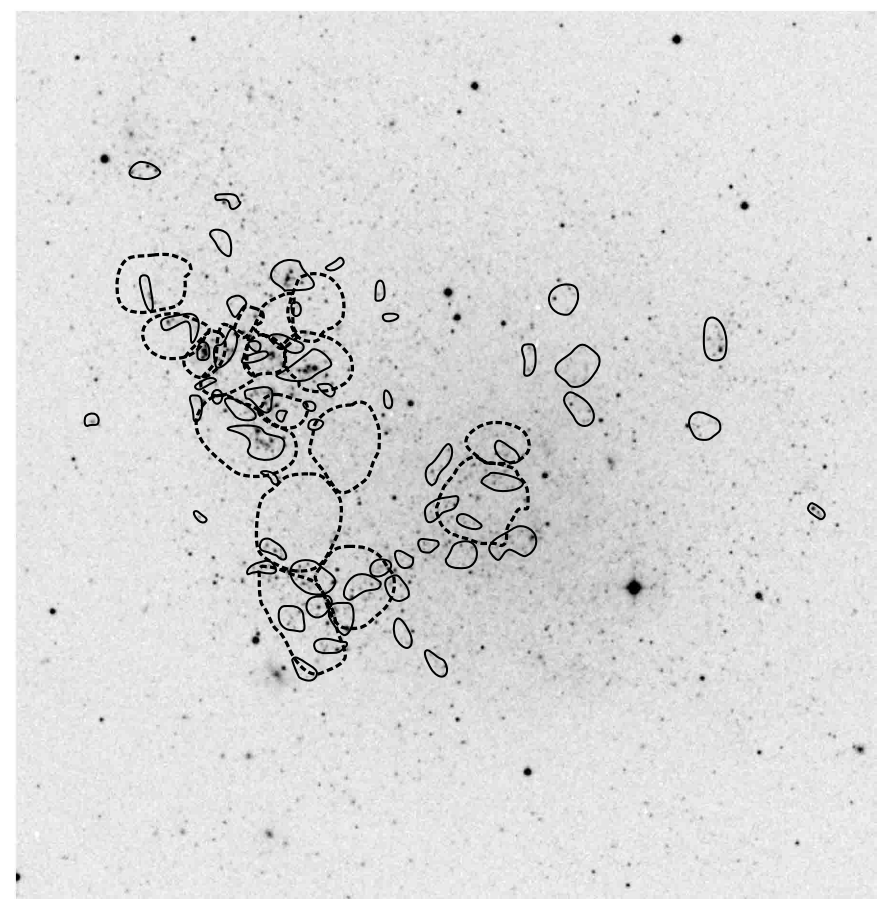

Fig. 3. Map of the associations in IC 1613 outlined by the Battinelli (1991) algorithm (solid lines) with superimposed boundaries of superbubbles (dashed lines).

and they are probably physically connected to the gas. The map of the associations with superimposed boundaries of the superbubbles is shown in Fig. 3.

Some of the newly identified stellar associations are found at the boundaries of the superbubbles and/or in the interaction zone between them. This indicates new star formation at 
the edges of superbubbles. Does this suggest, however, a scenario of self-induced star formation where the shocks that create the larger diameter superbubbles induce the formation of new stars compressing the ambient neutral or molecular gas? And are these more recently formed associations in turn creating new H II regions and bubbles at the periphery of the original superbubble? Lozinskaya et al. (2002) consider that the energy of the stellar wind from the associations is not enough to form the ionized shells and found additional, intense sources of stellar wind at their boundary - early-type supergiants and giants. Lozinskaya et al. (2003) studied in detail the structure and kinematics of the neutral and ionized gas components in the complex of star formation in the NE region. They identified three extended (300-350 pc) neutral shells with which the brightest ionized shells in the complex of star formation are associated. They also found evidence of the physical interaction between the H I and H II shells in the region of the chain of stars, early-type giants and supergiants, detected by Lozinskaya et al. (2002). Taking into account the relative positions and ages of the $\mathrm{H} \mathrm{I}$ and $\mathrm{H} \mathrm{II}$ shells and $\mathrm{OB}$ associations in the complex, they suggested sequential or triggered star formation in the expanding neutral shells. In addition to the three brightest and most prominent $\mathrm{H}$ I shells, they found supergiant arches and ring structures in the galaxy whose sizes are comparable to the gaseous-disk thickness and assumed this as a tracer of preceding starbursts in IC 1613. Many OB associations however have no superbubbles. The gas in these associations could be evaporated or perhaps the ionization flux from the stars is not enough to ionize them. In order to clarify these possibilities we are currently in the process of obtaining deeper photometry of the associations and performing spectral identification of the brightest blue stars. The results of such an analysis will be given in a forthcoming paper.

\section{Summary}

We have presented the results of a search for OB associations in the dwarf irregular Galaxy IC 1613. Application of the Battinelli (1991) method resulted in the detection of 60 OB associations with sizes between 30 and 130 pc. Numerical analysis indicates that between $3-5 \mathrm{OB}$ associations in the whole sample could be randomly concentrated OB stars. We detected the expected strong correlation between the spatial distributions of associations and H II regions in IC 1613.

Acknowledgements. J.B. is supported by FONDAP Center for Astrophysics grant number 150010003. A part of this work was performed while J.B. was a visiting astronomer in the UNAM, Mexico. The authors gratefully acknowledge the useful comments by an anonymous referee. This research is partially based on data from the ING Archive. L.G. acknowledges the financial support of the CONACyT No. 34422-E. J.B. thanks Marcio Catelan for his help. This research was supported in part by the Bulgarian National Science Foundation grant under contract No. F-812/1998 with the Bulgarian Ministry of Education and Sciences.

\section{References}

Aparicio, A., \& Gallart, C. 1995, AJ, 110, 2105

Battinelli, P. 1991, A\&A, 244, 69

Bresolin, F., Kennicutt, Jr., Ferrareze, L., et al. 1998, AJ, 116, 119

Borissova, J., Georgiev, L., Kurtev, R., et al. 2000, RMxAA, 36, 151

Dolphin, A., Saha, A., Skillman, E., et al. 2000, ApJ, 550, 554

Freedman, W. 1988, AJ, 96, 1248

Georgiev, L., Borissova, J., Rosado, M., et al. 1999, A\&AS, 134, 21

Hodge, P. 1978, ApJS, 37, 145

Ivanov, G. 1996, A\&A, 305, 708

Kontizas, E., Kontizas, M., Gouliermis, D., et al. 1999, in New view of the Magellanic Clouds, ed. Y.-H., Chu, N. Suntzeff, J. Hesser, \& D. Bohlender (San Francisco:ASP), IAU Symp., 190, 410

Landolt, A. 1992, AJ, 104, 340

Lejeune, T., \& Schaerer, D. 2001, A\&A, 365, 535

Lozinskaya, T., Arkhipova, V., Moiseev, A., \& Afanasiev, V. 2002, Astron. Rep., 46, 16

Lozinskaya, T., Moiseev, A., \& Podorvanyuk, N. 2003, Astron. Lett., 29,77

Lada, C., \& Lada, E. 2003, eprint arXiv [astro-ph/0301540]

Pietrzynski, G., Gieren, W., Fouque, P., \& Pont, F. 2001, A\&A, 371, 497

Rosado, M., Valdez-Gutierrez, M. M., Georgiev, L., et al. 2001, AJ, 122, 194

Stetson, P. 1993, Users manual for DAOPHOT II

Valdez-Gutierrez, M., Rosado, M., Georgiev, L., Borissova, J., \& Kurtev, R. 2001, A\&A, 366, 35 